\title{
Realizing the Hayden-Preskill protocol with coupled Dicke models
}

\author{
Yanting Cheng,,${ }^{1, *}$ Chang Liu $\odot,{ }^{1, *}$ Jinkang Guo $\odot,{ }^{2,1, *}$ Yu Chen, ${ }^{3}$ Pengfei Zhang,,${ }^{1,4,5}$ and Hui Zhai ${ }^{1, \dagger}$ \\ ${ }^{1}$ Institute for Advanced Study, Tsinghua University, Beijing 100084, China \\ ${ }^{2}$ School of Physics, Peking University, Beijing 100871, China \\ ${ }^{3}$ Center for Theoretical Physics, Department of Physics, Capital Normal University, Beijing 100048, China \\ ${ }^{4}$ Institute for Quantum Information and Matter, California Institute of Technology, Pasadena, California 91125, USA \\ ${ }^{5}$ Walter Burke Institute for Theoretical Physics, California Institute of Technology, Pasadena, California 91125, USA
}

(Received 22 October 2019; accepted 21 August 2020; published 5 October 2020)

\begin{abstract}
Hayden and Preskill proposed a thought experiment in which Bob can recover the information Alice throws into a black hole if he has a quantum computer entangled with the black hole, and for which Yoshida and Kitaev recently proposed a concrete decoding scheme. In the context of quantum many-body physics, the parallel question is that after a small system is thermalized with a large system, how can one decode the initial state information with the help of two entangled many-body systems? Here, we propose to realize this decoding protocol in a physical system of two Dicke models, with two cavity fields prepared in a thermofield double state. We show that the Yoshida-Kitaev protocol allows us to read out the initial spin information after it is scrambled into the cavity. We show that the readout efficiency reaches a maximum when the model parameters are tuned to the regime where the system is the most chaotic, characterized by the shortest scrambling time in the out-of-time-ordered correlation function. Our proposal opens up the possibility of discussing this profound thought experiment in a realistic setting.
\end{abstract}

DOI: 10.1103/PhysRevResearch.2.043024

\section{INTRODUCTION}

Quantum information scrambling now plays an important role in understanding quantum many-body systems. When a many-body system evolving from an initial state finally thermalizes, all the local information about the initial state gets lost, since a thermalized many-body system is described by only a few parameters such as temperature and chemical potential [1-4]. Precisely speaking, the local information of the initial state has been scrambled into the entire system during the process of quantum thermalization, such that the retrieval of this local information from local measurements is not possible [5,6]. This information loss is reminiscent of the black hole information problem. When Alice throws her diary into the black hole, Bob cannot recover the information in the diary from the Hawking radiation, which is just a small portion of the entire Hilbert space of a black hole. Here, the black hole is considered as the fastest information scrambler in our universe [7,8]. Recently, the out-of-time-ordered correlation (OTOC) function has been studied for describing the information scrambling processes, and a Lyapunov exponent can be defined to characterize the speed of information scrambling

\footnotetext{
*These authors contributed equally to this work.

†hzhai@tsinghua.edu.cn

Published by the American Physical Society under the terms of the Creative Commons Attribution 4.0 International license. Further distribution of this work must maintain attribution to the author(s) and the published article's title, journal citation, and DOI.
}

[8-14]. It is now known that a black hole possesses the largest Lyapunov exponent $[7,8]$.

In a seminal paper, Hayden and Preskill proposed a thought experiment for Bob to recover the information that Alice threw into a black hole, which is now known as the HaydenPreskill (HP) protocol [6]. The key of the HP protocol is to have another quantum system maximally entangled with the black hole and entirely under Bob's control. Based on general quantum information theory, Hayden and Preskill show that in this setting, it is possible for Bob to recover the information in Alice's diary by only manipulating the number of qubits much less than the total system [6]. In a parallel discussion of quantum many-body systems, it means that the initial state information can be recovered even after thermalization if two entangled many-body systems are prepared.

Recently, Yoshida and Kitaev (YK) went a step forward and described a procedure for realizing the HP protocol [15]. Their protocol requires the evolution of the quantum system described by a Haar random unitary evolution [16], which perfectly scrambles the information as a black hole. They also require that the unitary evolutions of two entangled many-body systems are conjugates of each other [15]. In this paper, we propose a concrete physical model for realizing the YK version of the HP protocol (hereafter referred to as the YKHP protocol) using two coupled Dicke models, as shown in Fig. 1(a). The quantum evolution of a physical system is governed by its Hamiltonian. Although thermalization and information scrambling can happen in most Hamiltonian systems except for a few exceptions such as many-body localization systems, most systems are not as chaotic as a Haar random, and the information scrambling in these systems is 
(a)

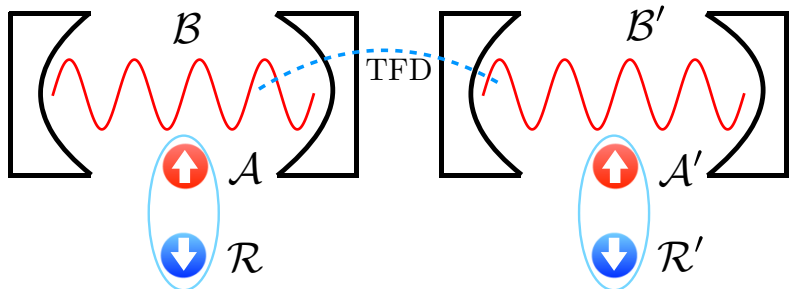

(b)

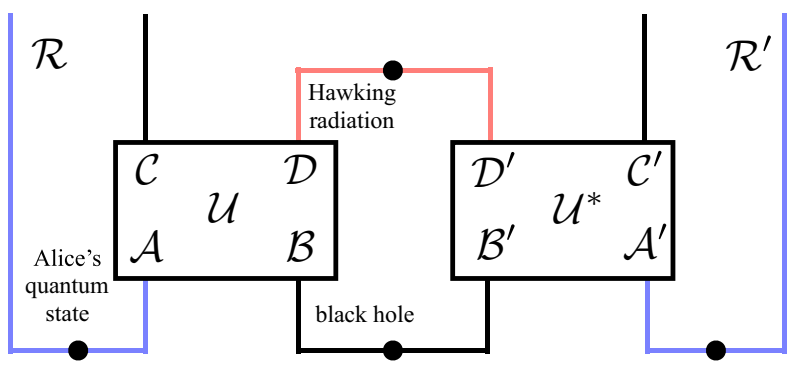

FIG. 1. (a) Schematic of our physical system of a coupled Dicke model, where TFD stands for the thermofield double state. (b) Diagrammatical illustration of the Yoshida and Kitaev version of the Hayden-Preskill protocol.

not as fast as a black hole. On the other hand, for two systems evolving under two conjugating unitary evolutions, the Hamiltonians for these two systems have to be opposite each other. Admittedly, in many synthetic quantum systems, the parameters in the Hamiltonian are largely tunable, so we should also anticipate that two Hamiltonians cannot be perfectly opposite each other [17-19]. These are major differences between the physical system and the ideal situation considered by YK. The purpose of this paper is to investigate how all these practical effects influence the efficiency of the YKHP protocol.

\section{YKHP PROTOCOL}

Before starting the discussion of our physical realization, let us first briefly review the YKHP protocol in Fig. 1(b) in Sec. II A, which is initially proposed for the infinitetemperature case [6,15], We will generalize the formalism to the finite-temperature case in Sec. II B, where we will show that the YKHP protocol also works for high but finite temperature.

\section{A. YKHP protocol at infinite temperature}

The YKHP protocol is shown in Fig. 1(b). In Fig. 1(b), $\mathcal{A}$ stands for a small quantum state with Hilbert space dimension $d_{\mathcal{A}}$ held by Alice, and $\mathcal{B}$ stands for a black hole as a large quantum system with Hilbert space dimension $d_{\mathcal{B}}$. $\mathcal{D}$ stands for the Hawking radiation as a small part of the black hole with Hilbert space $d_{\mathcal{D}}$. $\mathcal{C}$ stands for the remaining Hilbert space other than $\mathcal{D}$. The Hilbert space dimension of $\mathcal{C}$ is $d_{\mathcal{C}}$, and $d_{\mathcal{A}} d_{\mathcal{B}}=d_{\mathcal{C}} d_{\mathcal{D}}$. Their Hilbert space dimensions satisfy the condition $d_{\mathcal{A}} \ll d_{\mathcal{D}} \ll d_{\mathcal{B}}$. Here, throwing Alice's diary into the black hole means that the small system $\mathcal{A}$ is coupled to a large system $\mathcal{B}$, after which the total system undergoes a Haar random unitary evolution denoted by $\mathcal{U}$.

Before Alice couples her system $\mathcal{A}$ with the large system $\mathcal{B}$, she first backs up her information by forming an Einstein-
Podolsky-Rosen (EPR) pair between $\mathcal{A}$ and another reference system denoted by $\mathcal{R}$. Systems $\mathcal{R}$ and $\mathcal{A}$ have the same Hilbert space dimensions. Because system $\mathcal{R}$ is never coupled to the large system $\mathcal{B}$ and always remains independent during the evolution, and system $\mathcal{A}$ is coupled to $\mathcal{B}$, the EPR correlation between $\mathcal{A}$ and $\mathcal{R}$ smears out after thermalization. If without the right-half systems in Fig. 1(b) and Bob is allowed to perform measurements only on subsystem $\mathcal{D}$, it is not possible to recover Alice's information because it can be shown that the mutual information between $\mathcal{D}$ and $\mathcal{R}$ is strictly zero after the information is completely scrambled.

In this protocol, we need to introduce another half system denoted by $\mathcal{A}^{\prime}, \mathcal{B}^{\prime}, \mathcal{D}^{\prime}$, and $\mathcal{R}^{\prime}$, which have the same Hilbert space dimensions as $\mathcal{A}, \mathcal{B}, \mathcal{D}$, and $\mathcal{R}$, respectively. Initially, $\mathcal{B}$ and $\mathcal{B}^{\prime}$ form a maximally entangled state, and $\mathcal{A}^{\prime}$ and $\mathcal{R}^{\prime}$ form the same EPR correlation as that between $\mathcal{A}$ and $\mathcal{R}$. When $\mathcal{A}$ is coupled to $\mathcal{B}$, simultaneously $\mathcal{A}^{\prime}$ is also coupled to $\mathcal{B}^{\prime}$. The total system including $\mathcal{A}^{\prime}$ and $\mathcal{B}^{\prime}$ undergoes a unitary evolution $\mathcal{U}^{*}$ conjugating with $\mathcal{U}$. The essential point of YK's paper is that when $\mathcal{D}$ and $\mathcal{D}^{\prime}$ are projected to an EPR state, the initial EPR correlation will be recovered between $\mathcal{R}$ and $\mathcal{R}^{\prime}$. That is to say, let us use $\mathcal{F}$ to denote the conditional probability $\mathrm{P}\left(\mathrm{EPR}_{\mathcal{R R}^{\prime}} \mid \mathrm{EPR}_{\mathcal{D} \mathcal{D}^{\prime}}\right)$ for $\mathcal{R}$ and $\mathcal{R}^{\prime}$ being in an EPR state when $\mathcal{D}$ and $\mathcal{D}^{\prime}$ are projected to an EPR state, they show $\mathcal{F}=1$, when $\mathcal{U}$ and $\mathcal{U}^{*}$ are Haar random unitary and fully scramble the information. According to the Bayes' theorem, the conditional probability and the joint probability satisfy a relation $\mathrm{P}\left(\mathrm{EPR}_{\mathcal{R} \mathcal{R}^{\prime}}, \mathrm{EPR}_{\mathcal{D} \mathcal{D}^{\prime}}\right)=$ $\mathrm{P}\left(\mathrm{EPR}_{\mathcal{R R}^{\prime}} \mid \mathrm{EPR}_{\mathcal{D \mathcal { D } ^ { \prime }}}\right) \times \mathrm{P}\left(\mathrm{EPR}_{\mathcal{D} \mathcal{D}^{\prime}}\right)$, where $\mathrm{P}\left(\mathrm{EPR}_{\mathcal{D} \mathcal{D}^{\prime}}\right)$ is the probability of projecting a state into the $\mathrm{EPR}_{\mathcal{D D}}$ state and $\mathrm{P}\left(\mathrm{EPR}_{\mathcal{R R}^{\prime}}, \mathrm{EPR}_{\mathcal{D D}^{\prime}}\right)$ is the probability of projecting a state into the $\mathrm{EPR}_{\mathcal{D} \mathcal{D}^{\prime}}$ state and $\mathrm{EPR}_{\mathcal{R} \mathcal{R}^{\prime}}$ state simultaneously.

In order to calculate this conditional probability $\mathcal{F}$, we first calculate $\mathrm{P}\left(\mathrm{EPR}_{\mathcal{D \mathcal { D } ^ { \prime }}}\right)$ and $\mathrm{P}\left(\mathrm{EPR}_{\mathcal{R} \mathcal{R}^{\prime}}, \mathrm{EPR}_{\mathcal{D} \mathcal{D}^{\prime}}\right)$, respectively. Here, we first present some rules for the graphic calculation.

(1) Upward lines are ket vectors and downward lines are bra vectors. Connecting lines means summing all indices and the black dot on a line with the label $X$ is a overall factor $d_{X}^{-1 / 2}$ where $d_{X}$ represents the dimension of system $X$, for example,

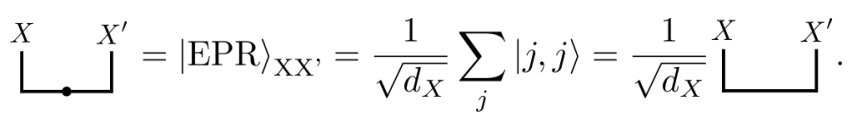

(2) Operators live in boxes. When a box is twisted upside down from one leg to another, the operator is changed from $X$ to $X^{T}$. For example,

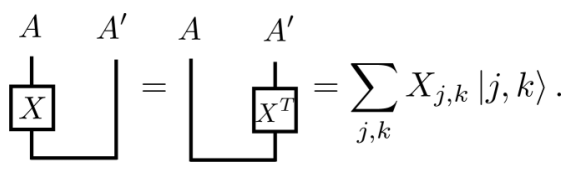

(3) The following is an important relation where

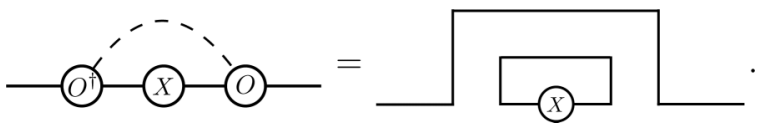

This equation means $\frac{1}{d_{X}^{2}} \sum_{O_{X} \in \mathbf{P}_{X}} O_{X}^{\dagger} X O_{X}=\frac{1}{d_{X}} \operatorname{Tr}(X) \equiv$ $\frac{1}{d_{X}} \sum_{j, k}\left\langle j\left|X_{j, k}\right| k\right\rangle \delta_{j, k}$, where $d_{X}$ denotes the dimension of this 
space, $O_{X}$ are generalized Pauli operators, $X$ is an arbitrary operator, and the dashed line represents an average over a complete set of all Pauli operators $\mathbf{P}_{X}$.

With these rules, we can proceed to calculate $\mathrm{P}\left(\mathrm{EPR}_{\mathcal{D} \mathcal{D}^{\prime}}\right)$ and $\mathrm{P}\left(\mathrm{EPR}_{\mathcal{R} \mathcal{R}^{\prime}}, \mathrm{EPR}_{\mathcal{D} \mathcal{D}^{\prime}}\right)$. In the YKHP protocal, initially,
$\mathcal{R}$ and $\mathcal{A}$ form an EPR state, $\mathcal{R}^{\prime}$ and $\mathcal{A}^{\prime}$ form an EPR state, and $\mathcal{B}$ and $\mathcal{B}^{\prime}$ form an EPR state. Then $\mathcal{A}$ and $\mathcal{B}$ together undergo a unitary evolution $\mathcal{U}$, and $\mathcal{A}^{\prime}$ and $\mathcal{B}^{\prime}$ together undergo a unitary evolution $\mathcal{U}^{*}$. Then, the state reads

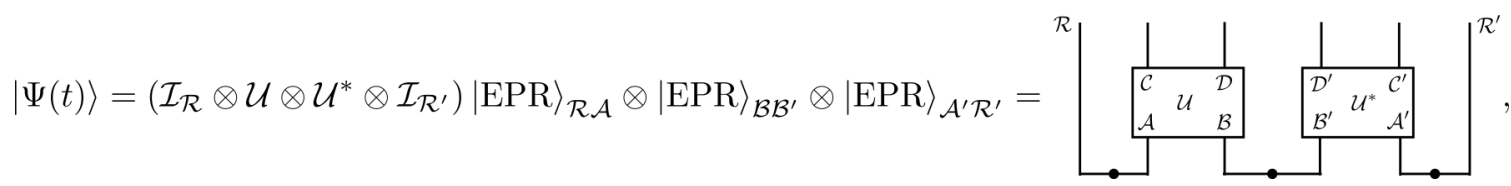

where $\mathcal{I}_{\mathcal{R}}$ and $\mathcal{I}_{\mathcal{R}}$ are identity matrices.

First, we calculate $\mathrm{P}\left(\mathrm{EPR}_{\mathcal{D} \mathcal{D}^{\prime}}\right)$. The projection operator onto an EPR state is graphically denoted by

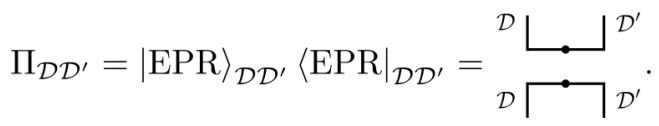

Thus $\mathrm{P}\left(\mathrm{EPR}_{\mathcal{D} \mathcal{D}^{\prime}}\right)$ reads

$$
\begin{aligned}
& \mathrm{P}\left(\mathrm{EPR}_{\mathcal{D} \mathcal{D}^{\prime}}\right)=\left\langle\Psi ( t ) \left|\left(\mathcal{I}_{\mathcal{R}} \otimes \mathcal{I}_{\mathcal{C}} \otimes|\mathrm{EPR}\rangle_{\mathcal{D D}^{\prime}}\left\langle\left.\mathrm{EPR}\right|_{\mathcal{D D}^{\prime}} \otimes \mathcal{I}_{\mathcal{C}^{\prime}} \otimes \mathcal{I}_{\mathcal{R}^{\prime}}\right)|\Psi(t)\rangle\right.\right.\right.
\end{aligned}
$$

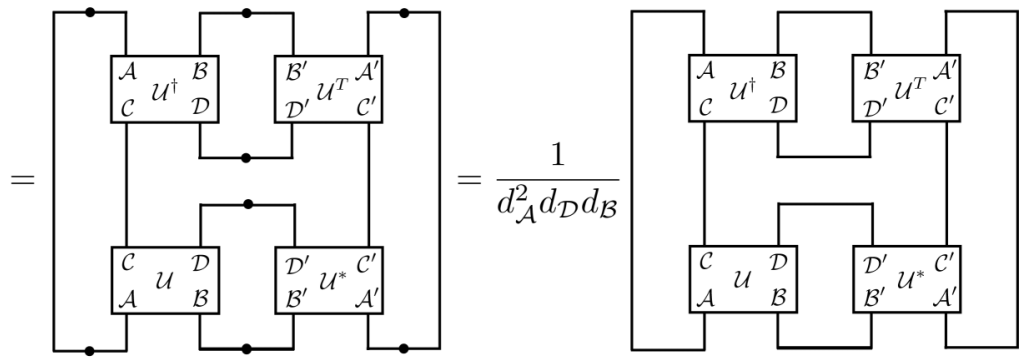

$$
\begin{aligned}
& =\frac{1}{d_{\mathcal{A}}^{2} d_{\mathcal{D}} d_{\mathcal{B}}} \\
& =\frac{1}{d_{\mathcal{A}}^{2} d_{\mathcal{D}} d_{\mathcal{B}}} \frac{d_{\mathcal{A}} d_{\mathcal{B}}}{d_{\mathcal{A}} d_{\mathcal{D}}} \sum_{\mathcal{O}_{\mathcal{A}} \in \mathbf{P}_{A}, \mathcal{O}_{\mathcal{D}} \in \mathbf{P}_{D}}\left\langle\mathcal{U}^{\dagger} \mathcal{O}_{\mathcal{D}}^{\dagger} \mathcal{U} \mathcal{O}_{\mathcal{A}}^{\dagger} \mathcal{U}^{\dagger} \mathcal{O}_{\mathcal{D}} \mathcal{U} \mathcal{O}_{\mathcal{A}}\right\rangle \\
& =\frac{1}{d_{\mathcal{A}}^{2} d_{\mathcal{D}}^{2}} \sum_{\mathcal{O}_{\mathcal{A}} \in \mathbf{P}_{\mathcal{A}}, \mathcal{O}_{\mathcal{D}} \in \mathbf{P}_{\mathcal{D}}}\left\langle\mathcal{O}_{\mathcal{D}}^{\dagger}(t) \mathcal{O}_{\mathcal{A}}^{\dagger} \mathcal{O}_{\mathcal{D}}(t) \mathcal{O}_{\mathcal{A}}\right\rangle,
\end{aligned}
$$

where $d_{\mathcal{A}}$ and $d_{\mathcal{D}}$ denote the dimension of space $\mathcal{A}$ and $\mathcal{D}$, respectively, and $\mathbf{P}_{\mathcal{A}}$ and $\mathbf{P}_{\mathcal{D}}$ denote the sets of all Pauli operators of space $\mathcal{A}$ and $\mathcal{D}$, respectively. In the first line we have used Eq. (5), and in the second line we have applied a combination of Eqs. (2) and (3) as the following equation,

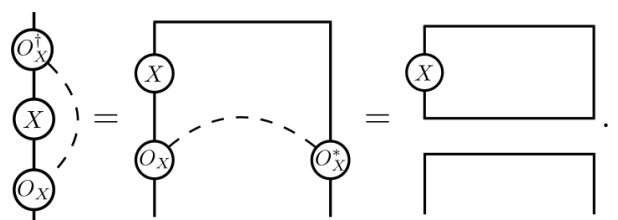


In the second equation of line 3 in Eq. (5), we have applied Eq. (2). Finally, we have defined $\langle\mathcal{O}\rangle=\left(1 / d_{\mathcal{O}}\right) \operatorname{Tr}(\mathcal{O})$.

Furthermore, if our system is a perfect scrambler, the time evolution operator can be regarded as a Haar random matrix. Then, one can use the property of a perfect scrambled system such that

$$
\begin{aligned}
& \langle A(t) X(0) B(t) Y(0)\rangle \\
& \quad \approx\langle A B\rangle\langle X\rangle\langle Y\rangle+\langle A\rangle\langle B\rangle\langle X Y\rangle-\langle A\rangle\langle B\rangle\langle X\rangle\langle Y\rangle
\end{aligned}
$$

to calculate $\mathrm{P}\left(\mathrm{EPR}_{\mathcal{D} \mathcal{D}^{\prime}}\right)$ as

$$
\begin{aligned}
\mathrm{P}\left(\mathrm{EPR}_{\mathcal{D} \mathcal{D}^{\prime}}\right) & =\frac{1}{d_{\mathcal{A}}^{2} d_{\mathcal{D}}^{2}} \sum_{\mathcal{O}_{\mathcal{A}} \subset \mathbf{P}_{\mathcal{A}}, \mathcal{O}_{\mathcal{D}} \subset \mathbf{P}_{\mathcal{D}}}\left\langle\mathcal{O}_{\mathcal{D}}^{\dagger}(t) \mathcal{O}_{\mathcal{A}}^{\dagger} \mathcal{O}_{\mathcal{D}}(t) \mathcal{O}_{\mathcal{A}}\right\rangle \\
& \approx \frac{1}{d_{\mathcal{A}}^{2} d_{\mathcal{D}}^{2}} \sum_{\mathcal{O}_{\mathcal{A}} \subset \mathbf{P}_{\mathcal{A}}, \mathcal{O}_{\mathcal{D}} \subset \mathbf{P}_{\mathcal{D}}}\left(\left\langle\mathcal{O}_{\mathcal{D}}^{\dagger} \mathcal{O}_{\mathcal{D}}\right\rangle\left\langle\mathcal{O}_{\mathcal{A}}^{\dagger}\right\rangle\left\langle\mathcal{O}_{\mathcal{A}}\right\rangle+\left\langle\mathcal{O}_{\mathcal{D}}^{\dagger}\right\rangle\left\langle\mathcal{O}_{\mathcal{D}}\right\rangle\left\langle\mathcal{O}_{\mathcal{A}}^{\dagger} \mathcal{O}_{\mathcal{A}}\right\rangle-\left\langle\mathcal{O}_{\mathcal{D}}^{\dagger}\right\rangle\left\langle\mathcal{O}_{\mathcal{D}}\right\rangle\left\langle\mathcal{O}_{\mathcal{A}}^{\dagger}\right\rangle\left\langle\mathcal{O}_{\mathcal{A}}\right\rangle\right) \\
& =\frac{1}{d_{\mathcal{A}}^{2} d_{\mathcal{D}}^{2}}\left(d_{\mathcal{D}}^{2}+d_{\mathcal{A}}^{2}-1\right) .
\end{aligned}
$$

Here, we have used the property that all Pauli matrices aside from identity are traceless $\langle O\rangle=0$. If $d_{D} \gg d_{A} \gg 1$, we have $\mathrm{P}\left(\mathrm{EPR}_{\mathcal{D} \mathcal{D}^{\prime}}\right) \approx 1 / d_{A}^{2}$.

Then, we proceed to compute the probability of simultaneously projecting the initial state to the EPR $\mathcal{D D}^{\prime}$ state and the EPR $\mathcal{R}^{\prime}$ state, which reads

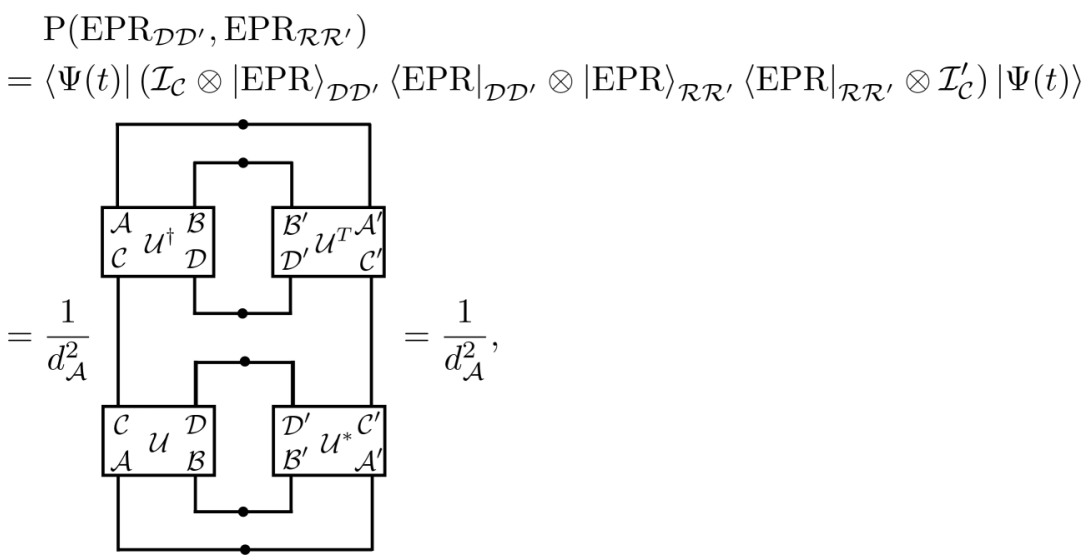

where $\mathcal{U}$ and $\mathcal{U}^{*}, \mathcal{U}^{\dagger}$ and $\mathcal{U}^{T}$ cancel out each other.

Then, by combining Eqs. (8) and (9), we obtain

$$
\begin{aligned}
\mathrm{P}\left(\mathrm{EPR}_{\mathcal{R} \mathcal{R}^{\prime}} \mid \mathrm{EPR}_{\mathcal{D} \mathcal{D}^{\prime}}\right) & =\frac{\mathrm{P}\left(\mathrm{EPR}_{\mathcal{D} \mathcal{D}^{\prime}}, \mathrm{EPR}_{\mathcal{R} \mathcal{R}^{\prime}}\right)}{\mathrm{P}\left(\mathrm{EPR}_{\mathcal{D} \mathcal{D}^{\prime}}\right)} \\
& =\frac{d_{\mathcal{D}}^{2}}{d_{\mathcal{D}}^{2}+d_{\mathcal{A}}^{2}-1} \approx 1,
\end{aligned}
$$

where we have again used the condition that the dimension of subsystem $\mathcal{D}$ is much larger than the dimension of subsystem $\mathcal{A}$.

\section{B. YKHP protocol at finite temperature}

Next, we will prove that the YK version of the HP protocol also works for a TFD state at finite temperature. We consider that systems $\mathcal{B}$ and $\mathcal{B}^{\prime}$ are prepared in a TFD state which is defined by

$$
\left|\mathrm{TFD}_{\mathcal{B} \mathcal{B}^{\prime}}\right\rangle=\frac{1}{\sqrt{Z}} \sum_{n} e^{-\beta E_{n} / 2}|n\rangle_{\mathrm{L}}|n\rangle_{\mathrm{R}},
$$


where $\beta=1 / k_{B} T, Z=\sum_{n} e^{-\beta E_{n}}$, and $|n\rangle_{\mathrm{L} / \mathrm{R}}$ denotes the eigenstate of the left/right system with energy $E_{n}$. Here, we present it graphically as $\left|\operatorname{TFD}_{\mathcal{B B}^{\prime}}\right\rangle=\left.{ }^{\mathcal{B}}\right|^{\mathcal{B}^{\prime}}$, where $\rho=e^{-\beta H} / Z$. We just modify Eqs. (5) and (9) as

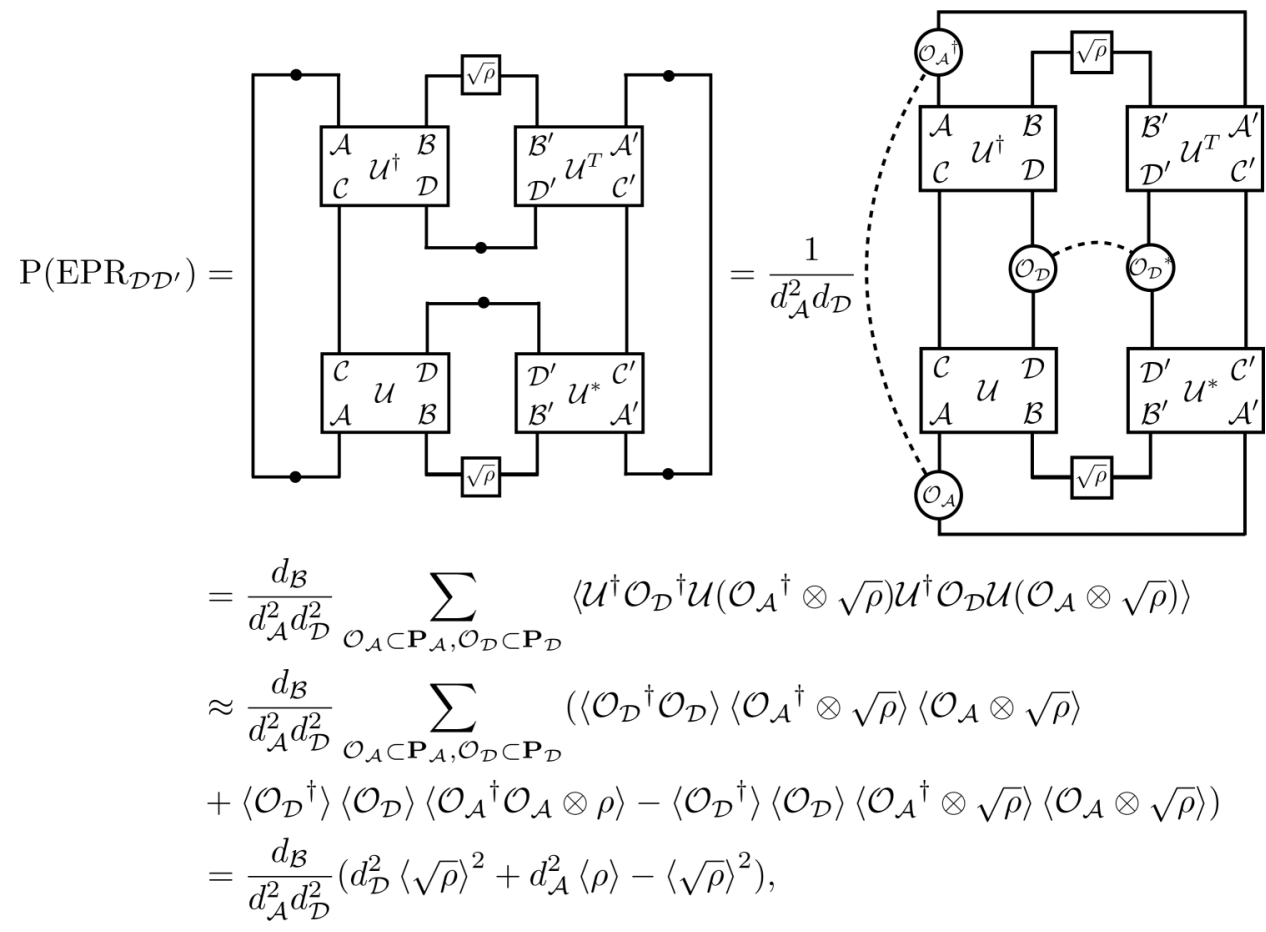

where $\langle\rho\rangle=\operatorname{Tr}(\hat{\rho}) / d_{\mathcal{B}},\langle\sqrt{\rho}\rangle=\operatorname{Tr}(\sqrt{\hat{\rho}}) / d_{\mathcal{B}}$. One can find that in the high-temperature limit $\beta \rightarrow 0,\langle\sqrt{\rho}\rangle=1 / \sqrt{d_{\mathcal{B}}}$, and $\langle\rho\rangle=$ $1 / d_{\mathcal{B}}$, therefore

$$
P\left(\mathrm{EPR}_{\mathcal{D} \mathcal{D}^{\prime}}\right)=\left(d_{\mathcal{D}}^{2}+d_{\mathcal{A}}^{2}-1\right) / d_{\mathcal{A}}^{2} d_{\mathcal{D}}^{2}
$$

For extremely low temperatures $\beta \rightarrow \infty,\langle\sqrt{\rho}\rangle=\langle\rho\rangle=1 / d_{\mathcal{B}}, P\left(\operatorname{EPR}_{\mathcal{D} \mathcal{D}^{\prime}}\right)=\left(d_{\mathcal{D}}^{2} / d_{\mathcal{B}}+d_{\mathcal{A}}^{2}-1 / d_{\mathcal{B}}\right) / d_{\mathcal{A}}^{2} d_{\mathcal{D}}^{2} \approx 1 / d_{\mathcal{D}}^{2}$

Similarly, for $\mathrm{P}\left(\mathrm{EPR}_{\mathcal{D} \mathcal{D}^{\prime}}, \mathrm{EPR}_{\mathcal{R} \mathcal{R}^{\prime}}\right)$, we have

$$
\begin{aligned}
& \mathrm{P}\left(\mathrm{EPR}_{\mathcal{D} \mathcal{D}^{\prime}}, \mathrm{EPR}_{\mathcal{R} \mathcal{R}^{\prime}}\right)=\frac{1}{d_{A}^{2}} \\
& =\frac{d_{\mathcal{B}}}{d_{\mathcal{A}}^{2} d_{\mathcal{D}}^{2}} \sum_{\mathcal{O}_{\mathcal{D} \subset \mathbf{P}_{\mathcal{D}}}}\left\langle\mathcal{U}^{\dagger} \mathcal{O}_{\mathcal{D}}^{\dagger} \mathcal{U}\left(\mathcal{I}_{\mathcal{A}} \otimes \sqrt{\rho}\right) \mathcal{U}^{\dagger} \mathcal{O}_{\mathcal{D}} \mathcal{U}\left(\mathcal{I}_{\mathcal{A}} \otimes \sqrt{\rho}\right)\right\rangle \\
& \approx \frac{d_{\mathcal{B}}}{d_{\mathcal{A}}^{2} d_{\mathcal{D}}^{2}} \sum_{\mathcal{O}_{\mathcal{D} \subset \mathbf{P}_{\mathcal{D}}}}\left(\left\langle\mathcal{O}_{\mathcal{D}}^{\dagger} \mathcal{O}_{\mathcal{D}}\right\rangle\left\langle\mathcal{I}_{\mathcal{A}} \otimes \sqrt{\rho}\right\rangle\left\langle\mathcal{I}_{\mathcal{A}} \otimes \sqrt{\rho}\right\rangle\right. \\
& \left.+\left\langle\mathcal{O}_{\mathcal{D}}{ }^{\dagger}\right\rangle\left\langle\mathcal{O}_{\mathcal{D}}\right\rangle\left\langle\mathcal{I}_{\mathcal{A}} \mathcal{I}_{\mathcal{A}} \otimes \rho\right\rangle-\left\langle\mathcal{O}_{\mathcal{D}}{ }^{\dagger}\right\rangle\left\langle\mathcal{O}_{\mathcal{D}}\right\rangle\left\langle\mathcal{I}_{\mathcal{A}} \otimes \sqrt{\rho}\right\rangle\left\langle\mathcal{I}_{\mathcal{A}} \otimes \sqrt{\rho}\right\rangle\right) \\
& =\frac{d_{\mathcal{B}}}{d_{\mathcal{A}}^{2} d_{\mathcal{D}}^{2}}\left(d_{\mathcal{D}}^{2}\langle\sqrt{\rho}\rangle^{2}+\langle\rho\rangle-\langle\sqrt{\rho}\rangle^{2}\right) \text {. }
\end{aligned}
$$


Finally, we obtain the result that

$$
\begin{aligned}
\mathrm{P}\left(\mathrm{EPR}_{\mathcal{R} \mathcal{R}^{\prime}} \mid \mathrm{EPR}_{\mathcal{D} \mathcal{D}^{\prime}}\right)= & \frac{\mathrm{P}\left(\mathrm{EPR}_{\mathcal{D} \mathcal{D}^{\prime}}, \mathrm{EPR}_{\mathcal{R} \mathcal{R}^{\prime}}\right)}{\mathrm{P}\left(\mathrm{EPR}_{\mathcal{D} \mathcal{D}^{\prime}}\right)} \\
= & \frac{d_{\mathcal{D}}^{2}\langle\sqrt{\rho}\rangle^{2}+\langle\rho\rangle-\langle\sqrt{\rho}\rangle^{2}}{d_{\mathcal{D}}^{2}\langle\sqrt{\rho}\rangle^{2}+d_{\mathcal{A}}^{2}\langle\rho\rangle-\langle\sqrt{\rho}\rangle^{2}} \\
& \approx \frac{d_{\mathcal{D}}^{2}\langle\sqrt{\rho}\rangle^{2} /\langle\rho\rangle+1}{d_{\mathcal{D}}^{2}\langle\sqrt{\rho}\rangle^{2} /\langle\rho\rangle+d_{\mathcal{A}}^{2}} .
\end{aligned}
$$

Here, we notice that

$$
\begin{aligned}
\frac{d_{\mathcal{D}}^{2}\langle\sqrt{\rho}\rangle^{2}}{d_{\mathcal{A}}^{2}\langle\rho\rangle} & =\frac{d_{\mathcal{D}}^{2}\left[\operatorname{Tr}\left(e^{-\beta \hat{H} / 2}\right)\right]^{2}}{d_{\mathcal{A}}^{2} d_{\mathcal{B}} \operatorname{Tr}\left(e^{-\beta \hat{H}}\right)}=\frac{d_{\mathcal{D}}^{2}}{d_{\mathcal{A}}^{2} d_{\mathcal{B}}} 2^{S^{(2)}(\beta / 2)} \\
& =2^{2 N_{\mathcal{D}}-2 N_{\mathcal{A}}+S^{(2)}(\beta / 2)-N_{\mathcal{B}}},
\end{aligned}
$$

where $N_{\mathcal{A}}, N_{\mathcal{B}}$, and $N_{\mathcal{D}}$ are numbers of qubits in $\mathcal{A}, \mathcal{B}$, and $\mathcal{D}$, such that $d_{\mathcal{A}}=2^{N_{\mathcal{A}}}, d_{\mathcal{B}}=2^{N_{\mathcal{B}}}$, and $d_{\mathcal{D}}=2^{N_{\mathcal{D}}}$. Here, $S^{(2)}(\beta / 2)$ is the second Rényi entropy [in units of $\left.\log (2)\right]$ of the thermal ensemble at inverse temperature $\beta / 2$. To derive the above equation, we have also used the fact that

$$
2^{-S^{(2)}(\beta / 2)}=\frac{\operatorname{Tr}\left(e^{-\beta \hat{H}}\right)}{\left[\operatorname{Tr}\left(e^{-\beta \hat{H} / 2}\right)\right]^{2}} .
$$

At high temperature, when $S^{(2)}(\beta / 2) \sim N_{\mathcal{B}}$, we have

$$
\frac{d_{\mathcal{D}}^{2}\langle\sqrt{\rho}\rangle^{2}}{d_{\mathcal{A}}^{2}\langle\rho\rangle} \gg 1 \text {. }
$$

Under this condition, the conditional probability $\mathrm{P}\left(\mathrm{EPR}_{\mathcal{R} \mathcal{R}^{\prime}} \mid \mathrm{EPR}_{\mathcal{D} \mathcal{D}^{\prime}}\right)$ can approach unity. However, for most models, $S^{(2)}(\beta / 2)$ approaches zero at low temperature, and given that $N_{\mathcal{D}}$ should be much smaller than half of $\mathcal{N}_{\mathrm{B}}$, therefore,

$$
\frac{d_{\mathcal{D}}^{2}\langle\sqrt{\rho}\rangle^{2}}{d_{\mathcal{A}}^{2}\langle\rho\rangle} \rightarrow 0
$$

Under this condition, we have $\mathrm{P}\left(\mathrm{EPR}_{\mathcal{R} \mathcal{R}^{\prime}} \mid \mathrm{EPR}_{\mathcal{D} \mathcal{D}^{\prime}}\right)=1 / \mathrm{d}_{\mathcal{A}}^{2}$. It means no information could be extracted. Here, the exception is the Sachdev-Ye-Kitaev $q$ (SYK- $q$ ) model with very large $q$, which has near-maximal extensive entropy even at zero temperature.

\section{COUPLED DICKE MODEL}

The Dicke model describes a spin coupled to a single-mode cavity photon field. The Hamiltonian for a single Dicke model is written as

$$
\hat{H}_{\text {Dicke }}(\hat{a}, \sigma)=\hbar \omega_{0} \hat{a}^{\dagger} \hat{a}+g\left(\hat{a}^{\dagger}+\hat{a}\right) \sigma_{x}+\hbar \omega_{z} \sigma_{z},
$$

where $\sigma$ is the Pauli matrices for a spin-1/2 and $\hat{a}$ is the cavity photon field, and we use $\hbar \omega_{0}$ as the unit of energy. We choose the Dicke model to realize the HP protocol for the following reasons.

First, the spin-1/2 plays the role of Alice's small system $\mathcal{A}$. The Hilbert space for the cavity field is the Fock space spanned by $\{|n\rangle, n=0, \ldots, \infty\}$, whose dimension is much larger than that of the Hilbert space $\mathcal{A}$, and the cavity field plays the role of the black hole $\mathcal{B}$. Suppose initially $g=0$ and the spin and the cavity are decoupled, then we turn on the coupling $g$ at $t=0$, and this quench process naturally mimics the processes that Alice's diary is thrown into the black hole. The quantum evolution under $\hat{H}_{\text {Dicke }}$ simulates $\mathcal{U}$.

Second, in $\hat{H}_{\text {Dicke }}, \hbar \omega_{0}$ is the cavity photon energy detuning compared with the background pumping laser field [20], $g$ is the cavity-atom coupling, and $\hbar \omega_{z}$ is the Zeeman field for a spin. All these parameters can be tuned over wide ranges and their signs can be changed. By inverting the signs of all three parameters, one can realize $-\hat{H}_{\text {Dicke }}$, under which the evolution realizes $\mathcal{U}^{*}$.

Third, one can generate a thermofield double (TFD) state between two cavity photons by a pair creation process or a two-mode squeezing process known in quantum optics [20-22]. Here, we consider two Dicke models with $\hat{H}=\hat{H}_{\mathrm{L}}+$ $\hat{H}_{\mathrm{R}}$, where $\hat{H}_{\mathrm{L}}=\hat{H}_{\text {Dicke }}\left(\hat{a}_{\mathrm{L}}, \sigma_{\mathrm{L}}\right)$ and $\hat{H}_{\mathrm{R}}=-\hat{H}_{\text {Dicke }}\left(\hat{a}_{\mathrm{R}}, \sigma_{\mathrm{R}}\right)$, where $\hat{a}_{\mathrm{L}}$ and $\hat{a}_{\mathrm{R}}$ are photon field operators for the left and the right cavity fields, and $\sigma_{\mathrm{L}}$ and $\sigma_{\mathrm{R}}$ are two spins-1/2 for $\mathcal{A}$ and $\mathcal{A}^{\prime}$. The Hamiltonian does not couple two sides, and the entanglement between two Dicke models is established through the initial state, which is prepared in a TFD state defined as $[23,24]$

$$
\left|\mathrm{TFD}_{\mathcal{B B}^{\prime}}\right\rangle=\frac{1}{\sqrt{Z}} \sum_{n} e^{-n \beta \hbar \omega_{0} / 2}|n\rangle_{\mathrm{L}}|n\rangle_{\mathrm{R}},
$$

where $Z=\sum_{n} e^{-\beta n \hbar \omega_{0}}$. The $\beta \rightarrow 0$ limit of the TFD state is the maximally entangled state used in YK's paper. Here, we always focus on the finite $\beta$ case, because the Hilbert space dimension for the cavity field is unbounded, and finite $\beta$ naturally introduces a cutoff such that our numerical calculation below can be safely done in a finite Hilbert space. It has been shown that YK's proposal also works for a TFD state at a small but finite $\beta$ in the previous section.

\section{THERMALIZATION AND OTOC}

We first consider a single Dicke model. Initially, we set $g=$ 0 where the spin and cavity are decoupled. We set that the cavity is in a thermal equilibrium state with $\beta=0.01$, and $\mathcal{A}$ and $\mathcal{R}$ are in a EPR state $\left|\Psi_{\mathcal{A R}}^{\mathrm{EPR}}\right\rangle=\frac{1}{\sqrt{2}}\left(|\uparrow\rangle_{\mathcal{A}}|\uparrow\rangle_{\mathcal{R}}+|\downarrow\rangle_{\mathcal{A}}|\downarrow\rangle_{\mathcal{R}}\right)$, which is one of the four Bell states. Then, at $t=0$ we turn on finite coupling $g$ and let the system evolve. For $\beta=0.01$ we considered here, we verified that it is safe to truncate the Hilbert space to several hundreds, and with this truncation we can numerically calculate the full quantum dynamics and obtain all correlation functions.

In Fig. 2(a), we show the projection of the wave function for the $\mathcal{A}-\mathcal{R}$ system into the four Bell states. One can see that as the system evolves, the populations on the four Bell states gradually approach $1 / 4$ equally. When all four populations become $1 / 4$, it indicates that the reduced density matrix for the spin $\mathcal{A}-\mathcal{R}$ subsystem is the identity. That is to say, all the initial state information about the spins is lost. Precisely speaking, the initial spin information is scrambled into the cavity field, which has a much larger Hilbert space dimension.

Here, we study the information scrambling in the Dicke model [25-27]. In Fig. 2(b), we calculate the OTOC between $\sigma_{x}$ and $\sigma_{z}$ for the same initial state. The timescale that OTOC decays to nearly zero defines the information scrambling time in a quantum many-body system. Strictly speaking, the 


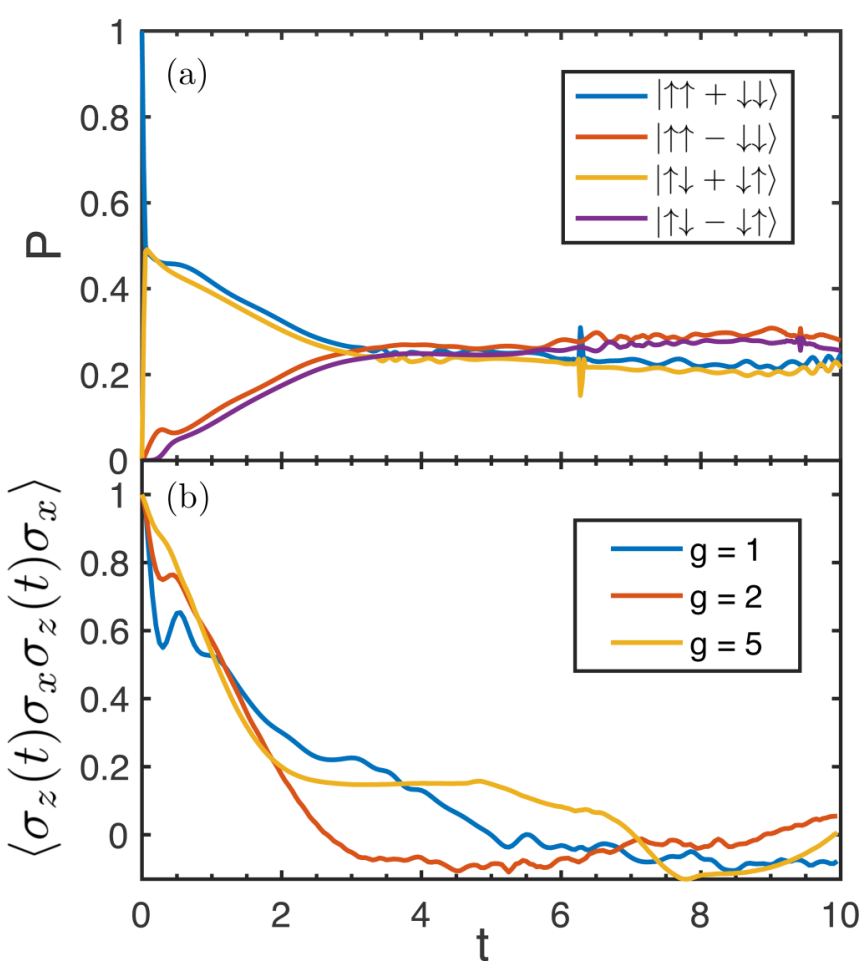

FIG. 2. Thermalization and OTOC for a single Dicke model. (a) The probability of projection onto the four Bell states formed by $\mathcal{A}$ and $\mathcal{R}$. (b) OTOC for the Dicke model with three different coupling constants $g / \hbar \omega_{0}$. Both (a) and (b) are plotted in terms of $t$ (in units of $\left.1 / \omega_{0}\right)$. Here, $\beta=0.01 / \hbar \omega_{0}$ and $\hbar \omega_{z}=3 \hbar \omega_{0}$.

information scrambling time is usually longer than the local thermalization time. However, since the Hilbert space for a single spin is quite small, it happens at nearly the same timescale. If one considers the OTOC between the spin and cavity field, the scrambling time is longer because it takes more time for spin information to be scrambled into the large Hilbert space of the cavity field.

Another notable feature in Fig. 2(b) is how the scrambling time depends on $g$. We show that the scrambling time for an intermediate $g$ is shorter than the scrambling times for both large $g$ and small $g$. This is because the system becomes close to integrable both in small and in large $g$ limits. At moderate $g$, the competition effect between the coupling term and other two terms is the most significant, which renders the system the most chaotic one in this regime. Similar results have been obtained for the Bose-Hubbard model [28] and for the spin model in a transverse field [29]. From the viewpoint of holographic duality, the regimes around the quantum critical point are most likely to have a holographic dual to a black hole, and therefore possess a larger Lyapunov exponent and a smaller scrambling time [28].

\section{EFFICIENCY OF THE YKHP PROTOCOL}

As discussed above, the key of the YKHP protocol is that when $\mathcal{D}$ and $\mathcal{D}^{\prime}$ are projected into an EPR state by measurement, $\mathcal{R}$ and $\mathcal{R}^{\prime}$ can recover the EPR correlation initially encoded between $\mathcal{A}$ and $\mathcal{R}$. Here, we will investigate how in

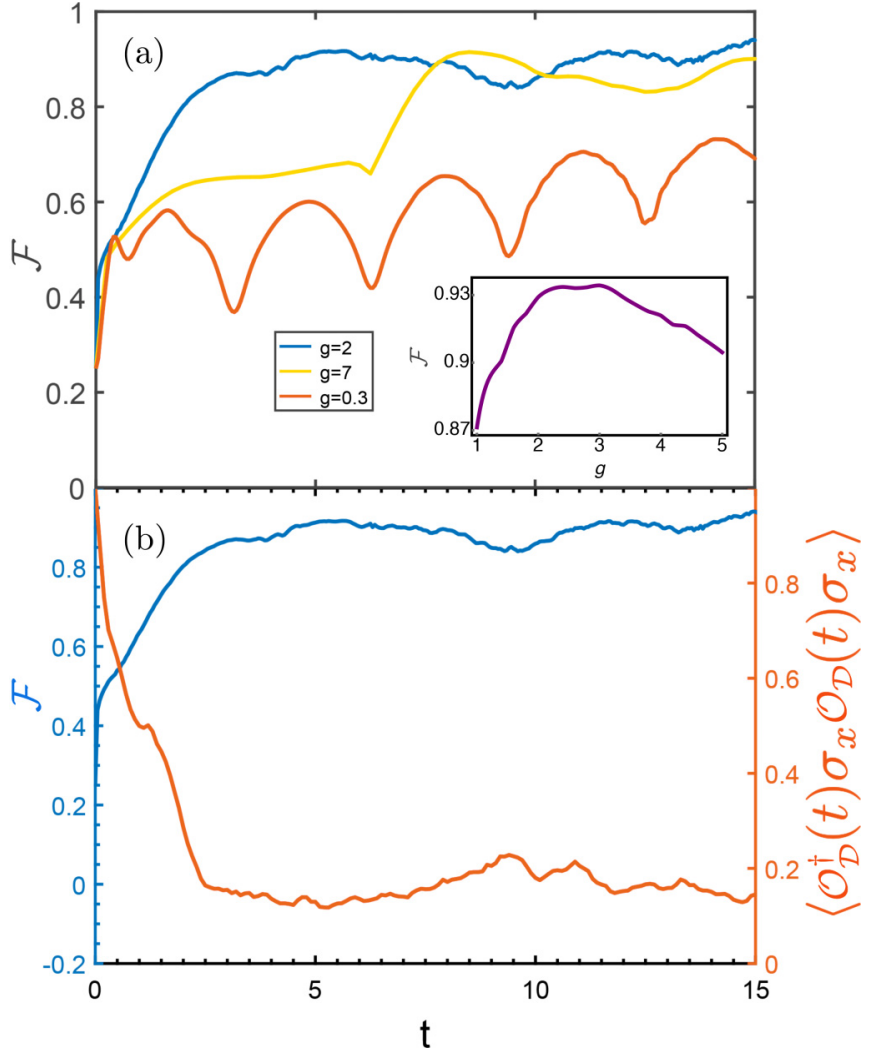

FIG. 3. (a) The conditional probability $\mathcal{F}$ as a function of $t$ for three different coupling constants $g$. (b) $\mathcal{F}$ and an OTOC between spin and an operator $\hat{\mathcal{O}}_{\mathcal{D}}$ in Hilbert space $\mathcal{D}$ are plotted in the same figure with the same coupling constant $g=2$.

our practical model, this conditional probability depends on time and coupling strength. Here, we need to first properly define the Hilbert space $\mathcal{D}$.

Considering a Fock state $|n\rangle_{\mathrm{L}}$ in the left cavity, we can formally write $|n\rangle_{\mathrm{L}}=\left|d_{\mathcal{D}} \times n_{\mathcal{C}}+n_{\mathcal{D}}\right\rangle_{\mathrm{L}} \equiv\left|n_{\mathcal{C}}, n_{\mathcal{D}}\right\rangle_{\mathrm{L}}$. Thus, we can define a $d_{\mathcal{D}}$-dimensional Hilbert space $\mathcal{D}$ as $\left\{\left|n_{\mathcal{D}}\right\rangle, n_{\mathcal{D}}=\right.$ $\left.0, \ldots, d_{\mathcal{D}}-1\right\}$. Here, we should take $d_{\mathcal{D}} \hbar \omega \ll k_{\mathrm{B}} T$ to ensure that $d_{\mathcal{D}}$ is much smaller than the dimension of occupied Hilbert space of $\mathcal{B}$. For example, take $d_{\mathcal{D}}=8$ when $k_{\mathrm{B}} T=$ 100 , so we can identify the Hilbert space $\mathcal{D}$ as a product of three pseudospins- $1 / 2$, denoted by $\tau_{\mathrm{L}}^{1}, \tau_{\mathrm{L}}^{2}$, and $\tau_{\mathrm{L}}^{3}$, and we can take $|0\rangle=|\uparrow\rangle_{\tau_{1}^{1}}|\uparrow\rangle_{\tau_{1}^{2}}|\uparrow\rangle_{\tau_{1}^{3}},|1\rangle=|\downarrow\rangle_{\tau_{L}^{1}}|\uparrow\rangle_{\tau_{1}^{2}}|\uparrow\rangle_{\tau_{L}^{3}}, \ldots$, $|7\rangle=|\downarrow\rangle_{\tau_{L}^{1}}|\downarrow\rangle_{\tau_{\perp}^{2}}|\downarrow\rangle_{\tau_{L}^{3}}$. Similarly, we can define $\mathcal{D}^{\prime}$ as a part of the right system, and introduce three pseudospins $\tau_{\mathrm{R}}^{1}, \tau_{\mathrm{R}}^{2}$, and $\tau_{\mathrm{R}}^{3}$ for $\mathcal{D}^{\prime}$. Therefore, the EPR state between $\mathcal{D}$ and $\mathcal{D}^{\prime}$ is defined as

$$
\left|\Psi_{\mathcal{D} \mathcal{D}^{\prime}}^{\mathrm{EPR}}\right\rangle=\prod_{i=1,2,3}\left(|\uparrow\rangle_{\tau_{\mathrm{L}}^{i}}|\uparrow\rangle_{\tau_{\mathrm{R}}^{i}}+|\downarrow\rangle_{\tau_{\mathrm{L}}^{i}}|\downarrow\rangle_{\tau_{\mathrm{R}}^{i}}\right) .
$$

Our physical simulation of YKHP protocol works as follows: (i) We start with the initial state as

$$
|\Phi(t=0)\rangle=\left|\Psi_{\mathcal{A R}}^{\mathrm{EPR}}\right\rangle \otimes\left|\Psi_{\mathcal{A}^{\prime} \mathcal{R}^{\prime}}^{\mathrm{EPR}}\right\rangle \otimes\left|\mathrm{TFD}_{\mathcal{B B}^{\prime}}\right\rangle,
$$


and (ii) we evolve the state with two Dicke Hamiltonians and reach $|\Phi(t)\rangle$. (iii) We project $|\Phi(t)\rangle$ onto the EPR state between $\mathcal{D}$ and $\mathcal{D}^{\prime}$ and the wave function on the remaining Hilbert space reads

$$
|\tilde{\Phi}(t)\rangle=\left\langle\Psi_{\mathcal{D D}^{\prime}}^{\mathrm{EPR}} \mid \Phi(t)\right\rangle,
$$

and we can further project the state onto the EPR state between $\mathcal{R}$ and $\mathcal{R}^{\prime}$ and the wave function of the rest of the $\mathcal{C}$ and $\mathcal{C}^{\prime}$ Hilbert space reads

$$
|\tilde{\tilde{\Phi}}(t)\rangle=\left\langle\Psi_{\mathcal{R} \mathcal{R}^{\prime}}^{\mathrm{EPR}} \mid \tilde{\Phi}(t)\right\rangle .
$$

(iv) We compute the conditional probability $\mathcal{F}$ as

$$
\mathcal{F}=\frac{\langle\tilde{\tilde{\Phi}}(t) \mid \tilde{\tilde{\Phi}}(t)\rangle}{\langle\tilde{\Phi}(t) \mid \tilde{\Phi}(t)\rangle} .
$$

The results of $\mathcal{F}$ are shown in Fig. 3(a), with the emphasis on how $\mathcal{F}$ depends on $g$. First, $\mathcal{F}$ increases with increasing time $t$, and it saturates at a long time. In Fig. 3(b) we compare the saturation time of $\mathcal{F}$ with the scrambling time of an OTOC between a spin operator and an operator $\hat{\mathcal{O}}_{\mathcal{D}}$ in the Hilbert space $\mathcal{D}$, defined as $\tau_{\mathrm{L}}^{1, x} \otimes \tau_{\mathrm{L}}^{2, y} \otimes \tau_{\mathrm{L}}^{3, z}$, and find that these two timescales are consistent [15]. Thus, as discussed above, the scrambling time is shorter for an intermediate $g$, and consequently, $\mathcal{F}$ also saturates in a relatively shorter timescale.
Second, the long-time saturation value of $\mathcal{F}$ also depends on $g$ nonmonotonically, as shown in the inset of Fig. 3(a). It reaches a maximum value of $\sim 0.93$ for $g$ of around $2-3$, where the system is in the most chaotic regime. That is the regime where the quantum evolution most closely resembles a Haar random unitary evolution.

\section{STABILITY OF THE YKHP PROTOCOL}

Now we consider the situation where the parameters in $\hat{H}_{\mathrm{L}}$ are not exactly the opposite of $\hat{H}_{\mathrm{R}}$, which was previously considered in Refs. [17,18]. Aside from the minus sign, either their coupling constant $g$ differs by $\delta g$ or the photon energy differs by $\delta \omega_{0}$. In Fig. 4 we show how the long-time saturation value of $\mathcal{F}$ depends on either $\delta g$ or $\delta \omega_{0}$. It shows that on one hand, $\mathcal{F}$ decreases from being close to unity to about $1 / 4$ with an increasing absolute value of either $\delta g$ or $\delta \omega_{0}$. Here, $1 / 4$ means that the EPR correlation between $\mathcal{R}$ and $\mathcal{R}^{\prime}$ is completely uncorrelated with the EPR correlation between $\mathcal{D}$ and $\mathcal{D}^{\prime}$. When the system is projected onto the EPR state of $\mathcal{D}$ and $\mathcal{D}^{\prime}$, the system has an equal probability of populating four Bell states of $\mathcal{R}$ and $\mathcal{R}^{\prime}$.

To quantify how fast the conditional probability $\mathcal{F}$ decays with the difference between two systems' Hamiltonians increasing, we respectively rewrite Eqs. (5) and (9) as

$$
\begin{aligned}
& \mathrm{P}\left(\mathrm{EPR}_{\mathcal{D} \mathcal{D}^{\prime}}\right)=\left\langle\Psi ( t ) \left|\left(\mathcal{I}_{\mathcal{R}} \otimes \mathcal{I}_{\mathcal{C}} \otimes|\mathrm{EPR}\rangle_{\mathcal{D D}^{\prime}}\left\langle\left.\mathrm{EPR}\right|_{\mathcal{D D}^{\prime}} \otimes \mathcal{I}_{\mathcal{C}^{\prime}} \otimes \mathcal{I}_{\mathcal{R}^{\prime}}\right)|\Psi(t)\rangle\right.\right.\right.
\end{aligned}
$$

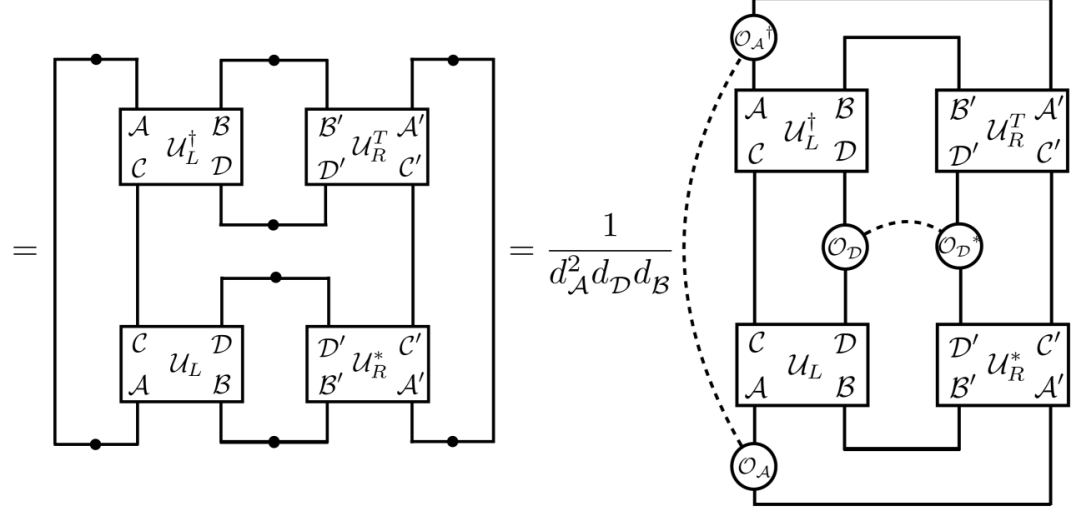

$$
\begin{aligned}
& =\frac{1}{d_{\mathcal{A}}^{2} d_{\mathcal{D}}^{2}} \sum_{\mathcal{O}_{\mathcal{A}} \subset \mathbf{P}_{\mathcal{A}}, O_{D} \subset \mathbf{P}_{\mathcal{D}}}\left\langle\mathcal{U}_{R}^{\dagger} \mathcal{O}_{\mathcal{D}}^{\dagger} \mathcal{U}_{R} \mathcal{O}_{\mathcal{A}}^{\dagger} \mathcal{U}_{L}^{\dagger} \mathcal{O}_{\mathcal{D}} \mathcal{U}_{L} \mathcal{O}_{\mathcal{A}}\right\rangle \\
& =\frac{1}{d_{\mathcal{A}}^{2} d_{\mathcal{D}}^{2}} \sum_{\mathcal{O}_{\mathcal{A}} \subset \mathbf{P}_{\mathcal{A}}, \mathcal{O}_{\mathcal{D}} \subset \mathbf{P}_{\mathcal{D}}}\left\langle\mathcal{U}_{L}^{\dagger} \mathcal{S O}_{\mathcal{D}}^{\dagger} \mathcal{S}^{\dagger} \mathcal{U}_{L} \mathcal{O}_{\mathcal{A}}^{\dagger} \mathcal{U}_{L}^{\dagger} \mathcal{O}_{\mathcal{D}} \mathcal{U}_{L} \mathcal{O}_{\mathcal{A}}\right\rangle \\
& \approx \frac{1}{d_{\mathcal{A}}^{2} d_{\mathcal{D}}^{2}} \sum_{\mathcal{O}_{\mathcal{A}} \subset \mathbf{P}_{\mathcal{A}}, \mathcal{O}_{\mathcal{D}} \subset \mathbf{P}_{\mathcal{D}}}\left(\left\langle\mathcal{S O}_{\mathcal{D}}^{\dagger} \mathcal{S}^{\dagger} \mathcal{O}_{\mathcal{D}}\right\rangle\left\langle\mathcal{O}_{\mathcal{A}}^{\dagger}\right\rangle\left\langle\mathcal{O}_{\mathcal{A}}\right\rangle+\left\langle\mathcal{O}_{\mathcal{D}}^{\dagger}\right\rangle\left\langle\mathcal{O}_{\mathcal{D}}\right\rangle\left\langle\mathcal{O}_{\mathcal{A}}^{\dagger} \mathcal{O}_{\mathcal{A}}\right\rangle-\left\langle\mathcal{O}_{\mathcal{D}}^{\dagger}\right\rangle\left\langle\mathcal{O}_{\mathcal{D}}\right\rangle\left\langle\mathcal{O}_{\mathcal{A}}^{\dagger}\right\rangle\left\langle\mathcal{O}_{\mathcal{A}}\right\rangle\right) \\
& =\frac{1}{d_{\mathcal{A}}^{2} d_{\mathcal{D}}^{2}} \sum_{\mathcal{O}_{\mathcal{D}} \subset \mathbf{P}_{\mathcal{D}}}\left(\left\langle\mathcal{S} \mathcal{O}_{\mathcal{D}}^{\dagger} \mathcal{S}^{\dagger} \mathcal{O}_{\mathcal{D}}\right\rangle+d_{\mathcal{A}}^{2}-1\right)
\end{aligned}
$$




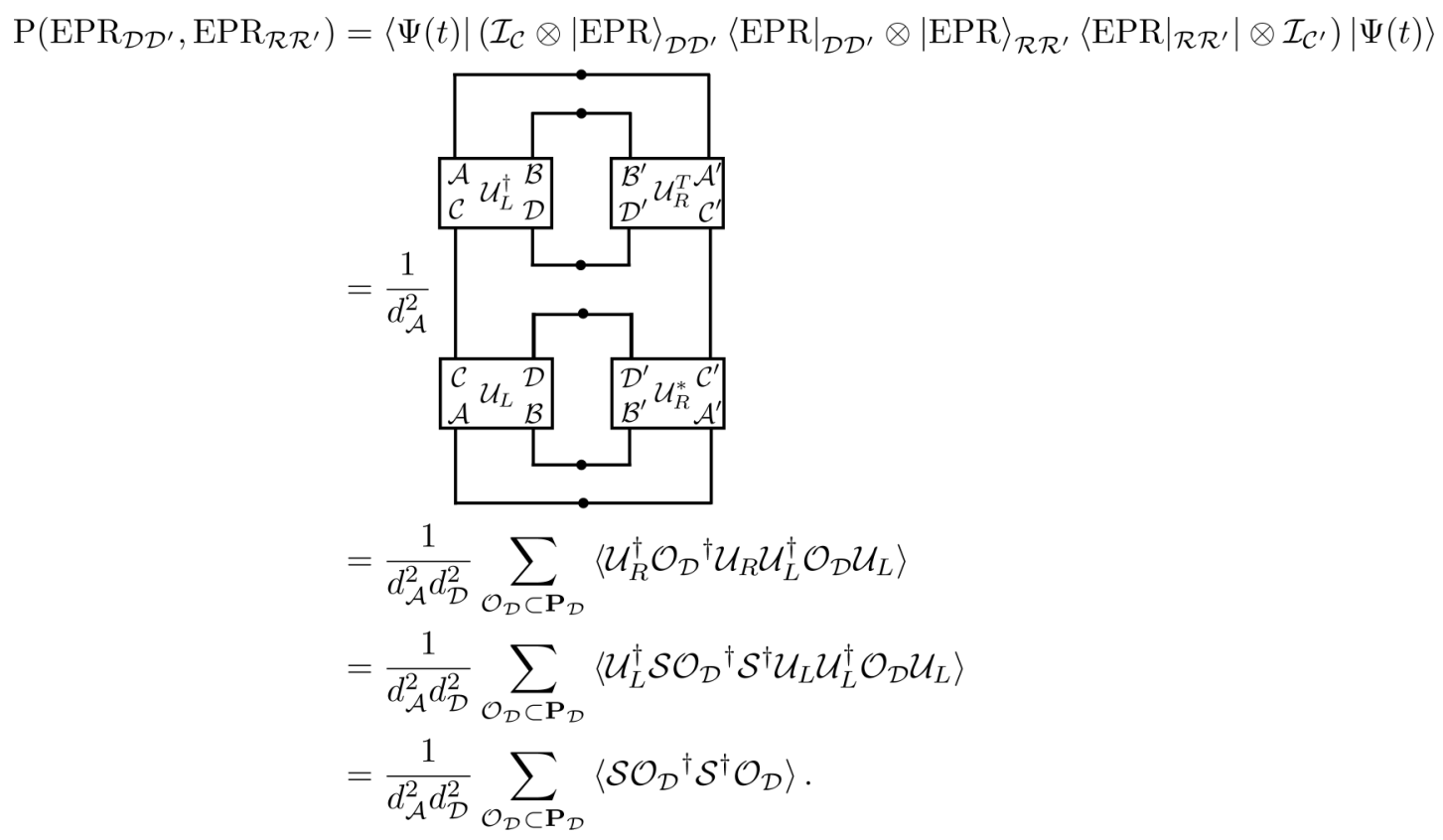

Here, $\hat{\mathcal{S}}$ denotes $\mathcal{U}_{\mathrm{L}} \mathcal{U}_{\mathrm{R}}^{\dagger}$, where $\mathcal{U}_{\mathrm{L}}$ denotes the evolution under $\hat{H}_{\mathrm{L}}$ and $\mathcal{U}_{\mathrm{R}}$ denotes the evolution under $\hat{H}_{\mathrm{R}}$. Finally, we obtain the conditional probability

$$
\begin{aligned}
\mathcal{F} & =\mathrm{P}\left(\mathrm{EPR}_{\mathcal{R R}^{\prime}} \mid \mathrm{EPR}_{\mathcal{D D}^{\prime}}\right) \\
& =\frac{\mathrm{P}\left(\mathrm{EPR}_{\mathcal{D D}^{\prime}}, \mathrm{EPR}_{\mathcal{R R}^{\prime}}\right)}{\mathrm{P}\left(\mathrm{EPR}_{\mathcal{D D}^{\prime}}\right)} \\
& =\frac{\sum_{\hat{\mathcal{O}}_{\mathcal{D}}}\left\langle\hat{\mathcal{O}}_{\mathcal{D}} \hat{\mathcal{S}} \hat{\mathcal{O}}_{\mathcal{D}}^{\dagger} \hat{\mathcal{S}}^{\dagger}\right\rangle}{d_{\mathcal{A}}^{2}-1+\sum_{\mathcal{O}_{\mathcal{D}}}\left\langle\hat{\mathcal{O}}_{\mathcal{D}} \hat{\mathcal{S}} \hat{\mathcal{O}}_{\mathcal{D}}^{\dagger} \hat{\mathcal{S}}^{\dagger}\right\rangle},
\end{aligned}
$$

where $d_{\mathcal{A}}$ is the Hilbert space dimension of system $\mathcal{A}$ and the summation over $\hat{\mathcal{O}}_{\mathcal{D}}$ runs over a complete set of operators in the Hilbert space $\mathcal{D}$. It is clear that if $\hat{H}_{\mathrm{R}}$ and $\hat{H}_{\mathrm{L}}$ are opposite each other, then $\mathcal{U}_{\mathrm{L}}=\mathcal{U}_{\mathrm{R}}$ and $\hat{\mathcal{S}}$ is the identity. Then $\sum_{\hat{\mathcal{O}}_{\mathcal{D}}}\left\langle\hat{\mathcal{O}}_{\mathcal{D}} \hat{\mathcal{O}}_{\mathcal{D}}^{\dagger}\right\rangle=d_{\mathcal{D}}^{2}$. Since $d_{\mathcal{D}} \gg d_{\mathcal{A}}$, we have $\mathcal{F} \rightarrow 1$. If $\hat{H}_{\mathrm{R}}$ and $\hat{H}_{\mathrm{L}}$ become two very different Hamiltonians, then $\hat{\mathcal{S}} \hat{\mathcal{O}}_{\mathcal{D}}^{\dagger} \hat{\mathcal{S}}^{\dagger}$ and $\hat{\mathcal{O}}_{\mathcal{D}}$ are two different operators, and $\left\langle\hat{\mathcal{O}}_{\mathcal{D}} \hat{\mathcal{S}} \hat{\mathcal{O}}_{\mathcal{D}}^{\dagger} \hat{\mathcal{S}}^{\dagger}\right\rangle$ vanishes except for $\hat{\mathcal{O}}_{\mathcal{D}}$ being the identity. Then $\mathcal{F}$ approaches $1 / d_{\mathcal{A}}^{2}$, in this case, since the $\mathcal{A}$ system is a single spin- $1 / 2$ and $d_{\mathcal{A}}=2$. That also explains why $\mathcal{F}$ approaches $1 / 4$ when the parameters of two Hamiltonians become very different.

\section{CONCLUSION}

In summary, we have presented a physical realization of the Hayden-Preskill protocol in two copies of Dicke models with the help of the thermofield double state. It is interesting to note the dual role of information scrambling regarding decoding the initial state information. In a single system, it is the information scrambling that prevents decoding the initial state information from local measurements after thermalization. But in the thermofield double system, it is also the information scrambling that allows the YKHP protocol to read out
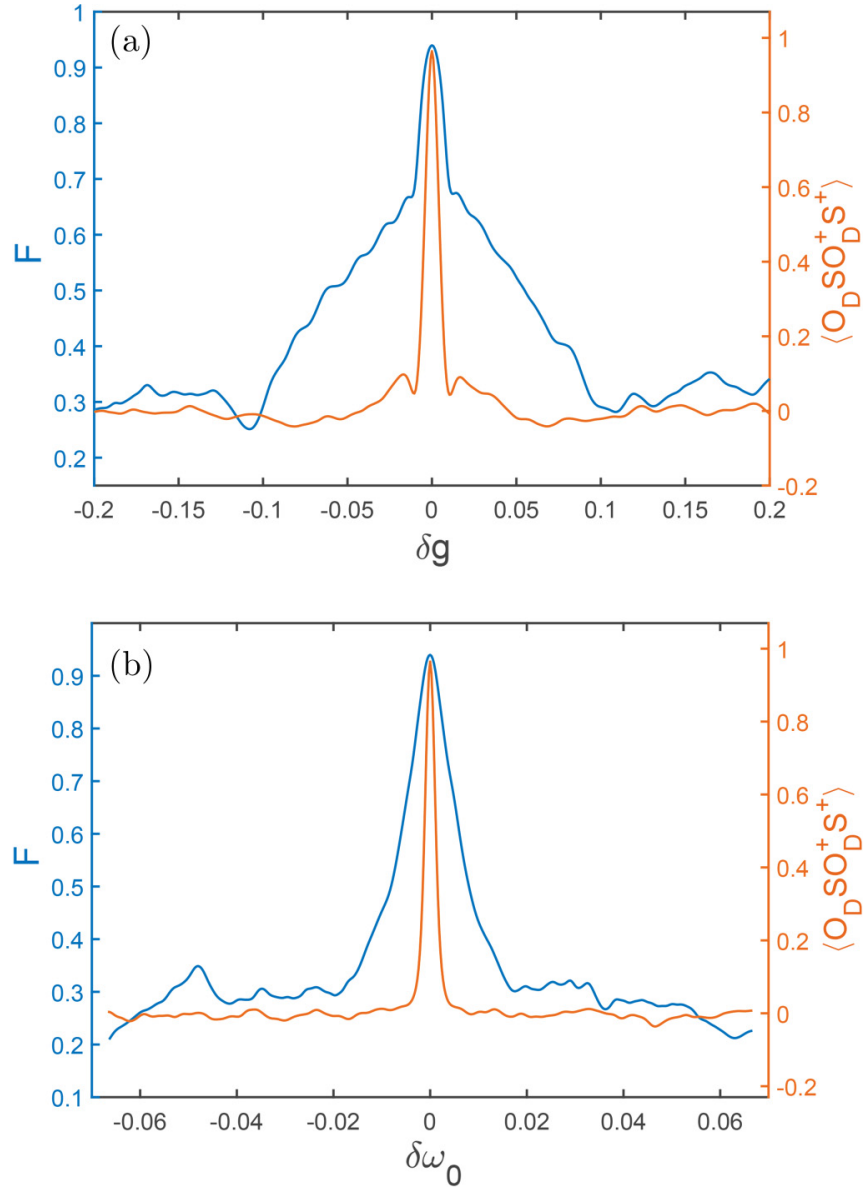

FIG. 4. The conditional probability $\mathcal{F}$ for (a) $\delta g$ and (b) $\delta \omega_{0}$, where $\delta g$ and $\delta \omega_{0}$ is the difference in coupling constant and difference in cavity photon frequency, respectively. We also plot the correlation function $\left\langle\hat{\mathcal{O}}_{\mathcal{D}} \hat{\mathcal{S}} \hat{\mathcal{O}}_{\mathcal{D}}^{\dagger} \hat{\mathcal{S}}^{\dagger}\right\rangle$ that is closely related to this dependence. 
the initial state information. We show that in our model, the decoding efficiency reaches a maximum close to unity for intermediate spin-cavity coupling $g$, where the system is most chaotic and the scrambling is the fastest. Our approach can also be straightforwardly applied to other Hamiltonian systems. We hope that such studies can simulate experimental simulations of the HP thought experiment in synthetic quantum systems. Our numerical simulations so far are limited to the teleportation of a single qubit and without including decoherence, due to the increasing computational complicity. A real experimental realization will enable us to study important issues, such as how teleportation efficiency and cost scale change with increasing qubit numbers and what are the effects of decoherence.

\section{ACKNOWLEDGMENTS}

This work is supported by Beijing Outstanding Young Scientist Program (H.Z.), MOST under Grant No. 2016YFA0301600 (H.Z.) and NSFC Grants No. 11734010 (H.Z. and Y.C.) and No. 11604225 (Y.C.), and Beijing Natural Science Foundation (Z180013) (Y.C.). P.Z. acknowledges support from the Walter Burke Institute for Theoretical Physics at Caltech.
[1] J. M. Deutsch, Quantum statistical mechanics in a closed system, Phys. Rev. A 43, 2046 (1991).

[2] M. Srednicki, Chaos and quantum thermalization, Phys. Rev. E 50, 888 (1994).

[3] M. Rigol, V. Dunjko, and M. Olshanii. Thermalization and its mechanism for generic isolated quantum systems, Nature (London) 452, 854 (2008).

[4] A. M. Kaufman, M. E. Tai, A. Lukin, M. Rispoli, R. Schittko, P. M. Preiss, and M. Greiner, Quantum thermalization through entanglement in an isolated many-body system, Science $\mathbf{3 5 3}$, 794 (2016).

[5] D. N. Page, Expected Entropy of a Subsystem, Phys. Rev. Lett. 71, 1291 (1993).

[6] P. Hayden and J. Preskill, Black holes as mirrors: Quantum information in random subsystems, J. High Energy Phys. 09 (2007) 120.

[7] J. Maldacena, S. H. Shenker, and D. Stanford, A bound on chaos, J. High Energy Phys. 08 (2016) 106.

[8] S. H. Shenker and D. Stanford, Black holes and the butterfly effect, J. High Energy Phys. 03 (2014) 067.

[9] A. I. Larkin and Y. N. Ovchinnikov, Quasiclassical method in the theory of superconductivity, Sov. Phys. JETP 28, 1200 (1969).

[10] A. Kitaev, Hidden correlations in the Hawking radiation and thermal noise, a talk given at Fundamental Physics Prize Symposium, 2014, https://www.on.kitp.ucsb.edu/online/ joint98/kitaev/.

[11] P. Hosur, X. L. Qi, D. A. Roberts, and B. Yoshida, Chaos in quantum channels, J. High Energy Phys. 02 (2016) 004

[12] R. Fan, P. Zhang, H. Shen, and H. Zhai, Out-of-time-order correlation for many-body localization, Sci. Bull. 62, 707 (2017).

[13] J. Li, R. Fan, H. Wang, B. Ye, B. Zeng, H. Zhai, X. Peng, and J. Du, Measuring Out-of-Time-Order Correlators on a Nuclear Magnetic Resonance Quantum Simulator, Phys. Rev. X 7, 031011 (2017).

[14] M. Gärttner, J. G. Bohnet, A. Safavi-Naini, M. L. Wall, J. J. Bollinger, and A. M. Rey, Measuring out-of-time-order correlations and multiple quantum spectra in a trapped-ion quantum magnet, Nat. Phys. 13, 781 (2017).
[15] B. Yoshida and A. Kitaev, Efficient decoding for the HaydenPreskill protocol, arXiv:1710.03363.

[16] Y. Sekino and L. Susskind, Fast scramblers, J. High Energy Phys. 10 (2008) 065.

[17] B. Yoshida and N. Y. Yao, Disentangling Scrambling and Decoherence via Quantum Teleportation, Phys. Rev. X 9, 011006 (2019).

[18] K. A. Landsman et al., Verified quantum information scrambling, Nature (London) 567, 61 (2019).

[19] B. Swingle and N. Yunger Halpern, Phys. Rev. A 97, 062113 (2018).

[20] M. O. Scully and M. S. Zubairy, Quantum Optics (Cambridge University Press, Cambridge, U.K., 1999).

[21] B. L. Schumaker and C. M. Caves, New formalism for two-photon quantum optics. II. Mathematical foundation and compact notation, Phys. Rev. A 31, 3093 (1985).

[22] J. Hu, L. Feng, Z. Zhang, and C. Chin, Quantum simulation of coherent Hawking-Unruh radiation, Nat. Phys. 15, 785 (2019).

[23] W. Israel, Thermo-field dynamics of black holes, Phys. Lett. A 57, 107 (1976).

[24] J. Maldacena, Eternal black holes in anti-de Sitter, J. High Energy Phys. 04 (2003) 021.

[25] Y. Alavirad and A. Lavasani, Scrambling in the Dicke model, Phys. Rev. A 99, 043602 (2019).

[26] R. J. Lewis-Swan, A. Safavi-Naini, J. J. Bollinger, and A. M Rey, Unifying fast scrambling, thermalization and entanglement through the measurement of FOTOCs in the Dicke model, Nat. Commun. 10, 1581 (2019).

[27] Q. Zhuang, T. Schuster, B. Yoshida, and N. Y. Yao, Scrambling and complexity in phase space, Phys. Rev. A 99, 062334 (2019).

[28] H. Shen, P. Zhang, R. Fan, and H. Zhai, Out-of-time-order correlation at a quantum phase transition, Phys. Rev. B 96, 054503 (2017)

[29] K. X. Wei, P. Peng, O. Shtanko, I. Marvian, S. Lloyd, C. Ramanathan, and P. Cappellaro, Emergent Prethermalization Signatures in Out-Of-Time Ordered Correlations, Phys. Rev. Lett. 123, 090605 (2019). 